\title{
Downregulation of miR-205 and miR-31 confers resistance to chemotherapy-induced apoptosis in prostate cancer cells
}

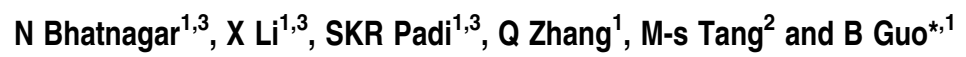

Advanced prostate cancers are known to acquire not only invasive capabilities but also significant resistance to chemotherapyinduced apoptosis. To understand how microRNAs (miRNAs) may contribute to prostate cancer resistance to apoptosis, we compared microRNA expression profiles of a benign prostate cancer cell line WPE1-NA22 and a highly malignant WPE1-NB26 cell line (derived from a common lineage). We found that miR-205 and miR-31 are significantly downregulated in WPE1-NB26 cells, as well as in other cell lines representing advanced-stage prostate cancers. Antiapoptotic genes BCL2L2 (encoding Bcl-w) and E2F6 are identified as the targets of miR-205 and miR-31, respectively. By downregulating Bcl-w and E2F6, miR-205 and miR-31 promote chemotherapeutic agents-induced apoptosis in prostate cancer cells. The promoter region of the miR-205 gene was cloned and was found to be hypermethylated in cell lines derived from advanced prostate cancers, contributing to the downregulation of the gene. Treatment with DNA methylation inhibitor 5-aza-2'-deoxycytidine induced miR-205 expression, downregulated Bcl-w, and sensitized prostate cancer cells to chemotherapy-induced apoptosis. Thus, downregulation of miR-205 and miR-31 has an important role in apoptosis resistance in advanced prostate cancer.

Cell Death and Disease (2010) 1, e105; doi:10.1038/cddis.2010.85; published online 9 December 2010

Subject Category: Cancer

Prostate cancer is one of the most common types of cancer and the second leading cause of cancer death in men (American Cancer Society). Surgery, radiation therapy, and hormone therapy are generally effective for early and localized cancers. However, as the prostate cancers progress to advanced stages and metastasize, chemotherapy is the only option and often fails to produce clinical response. It is known that as the prostate cancers evolve from the benign stage to more aggressive stages, they become more resistant to apoptosis. ${ }^{1-5}$ The resistance to apoptosis has been associated with increased expression of antiapoptotic proteins $\mathrm{Bcl}-2$, survivin, XIAP, and the multidrug resistanceassociated protein, as well as decreased expression of the pro-apoptotic proteins Bax and Bak. The exact mechanism of how advanced prostate cancers evade apoptosis remains poorly defined.

microRNAs (miRNAs) are small 19-25 nucleotide-long, single-stranded non-coding RNAs that silence target genes by cleaving mRNA molecules or inhibiting translation. ${ }^{6}$ The miRNAs are transcribed from their genes by pol II as long pri-miRNAs and processed into $\sim 60-70$ nucleotide-long pre-miRNA by Drosha RNase III endonuclease. ${ }^{7}$ Another RNase III endonuclease Dicer further processes the pre-miRNAs and the mature single-strand miRNAs are incorporated into the RNA-induced silencing complex (RISC) to recognize and bind to target mRNAs. Recently, miRNAs have been linked to cancer pathogenesis, regulating cell cycle, ${ }^{8}$ differentiation, ${ }^{9,10}$ metabolism, ${ }^{11}$ invasion and metastasis, ${ }^{12}$ as well as apoptosis. ${ }^{13}$

In cancer cells, tumor suppressor genes (RB1, CDKN2A, CDKN1A, CDH1, GSTP1, and so on.) can be silenced through hypermethylation of the $\mathrm{CpG}$ islands within the promoter region. ${ }^{14}$ DNA methylation results in the recruitment of proteins containing the methyl-CpG binding domain, which in turn attract histone deacetylases and histone methyltransferases to modify histones and inhibit gene expression. ${ }^{15}$ More than 40 genes have been shown to be epigenetically silenced by DNA methylation in prostate cancer. ${ }^{16}$ More interestingly, hypermethylation of the promoters of $\mathrm{ER} \alpha$, $\mathrm{hMLH} 1$, and p14/INK4a often occurs in advanced prostate cancers. ${ }^{17}$ Recently, a number of tumor suppressing miRNAs have been shown to be downregulated in cancer cells by $\mathrm{CpG}$ island methylation-associated silencing. ${ }^{18-23}$ Thus, epigenetic silencing of the tumor suppressing miRNAs may contribute as a novel mechanism for cancer development.

WPE1-NA22 and WPE1-NB26 are prostate cancer cell lines derived from immortalized human prostate epithelial RWPE- 1 cells by treatment with $N$-methyl- $N$-nitrosourea and sequential xenografts in nude mice. ${ }^{24}$ Although the WPE1-NA22 cells form small, non-invasive, well-differentiated tumors in nude mice, the WPE1-NB26 cells are highly malignant and form large, poorly differentiated, invasive

\footnotetext{
${ }^{1}$ Department of Pharmaceutical Sciences, College of Pharmacy, North Dakota State University, Fargo, ND, USA and ${ }^{2}$ Department of Environmental Medicine, New York University School of Medicine, Tuxedo, NY, USA

${ }^{*}$ Corresponding author: B Guo, Department of Pharmaceutical Sciences, College of Pharmacy, North Dakota State University, Fargo, ND 58108, USA.

Tel: 701-231-5164; Fax: 701-231-8333; E-mail: Bin.Guo@ndsu.edu

${ }^{3}$ These authors contributed equally to this work.

Keywords: miR-205; miR-31; E2F6; Bcl-w; apoptosis; prostate cancer

Abbreviations: miRNA, microRNA; $3^{\prime} U T R, 3^{\prime}$ untranslated region; $5^{\prime}$ RACE, Rapid amplification of $5^{\prime}$ complementary DNA ends; GFP, green fluorescent protein

Received 14.6.10; revised 19.10.10; accepted 28.10.10; Edited by G Melino
} 
tumors. We found that WPE1-NB26 cells were highly resistant to apoptosis induced by various agents, whereas WPE1NA22 cells were sensitive. By comparing the miRNA expression profiles between the two cell lines, we found that miR-205 and miR-31 were significantly downregulated in WPE1-NB26 cells. Downregulation of the two miRNAs resulted in upregulation of antiapoptotic proteins $\mathrm{Bcl}-\mathrm{w}$ and E2F6 and resistance to apoptosis. We further demonstrated that promoter hypermethylation of the miR-205 gene was responsible for its downregulation in advanced prostate cancer cells.

\section{Results}

WPE1-NB26 cells are resistant to various apoptosis stimuli. We compared the WPE1-NA22 (early cancer) and WPE1-NB26 (advanced cancer) prostate cancer cell lines for their responses to various apoptosis-inducing treatments. As shown in Figure 1, WPE1-NB26 cells were significantly more resistant to apoptosis induced by UV irradiation, $\mathrm{H}_{2} \mathrm{O}_{2}$, and chemotherapeutic agents Docetaxel and Cisplatin, comparing with the WPE1-NA22 cells. Induction of apoptosis was determined by the cell death ELISA assay measuring mono and oligonucleosomes in the lysates of apoptotic cells. The different apoptotic responses between the two cell lines were also confirmed by annexin $\mathrm{V}$ staining (Supplementary Figure $1 \mathrm{~A})$. To understand the mechanism that is responsible for the apoptosis resistance in WPE1-NB26 cells, we examined the expression of several apoptosis regulatory proteins (including $\mathrm{Bcl}-\mathrm{xL}, \mathrm{Mcl}-1, \mathrm{XIAP}$, Bid, and Bax) in the two cell lines. However, we did not observe a change in the levels of these apoptosis regulators that can explain the observed resistance in WPE1-NB26 cells. In fact, the levels of both antiapoptotic proteins Mcl-1 and XIAP were decreased in WPE1-NB26 cells, comparing with those in WPE1-NA22 cells (Supplementary Figure 1B).

miR-205 and miR-31 are downregulated in WPE1-NB26 cells. To determine whether differential miRNA expression has a role in the apoptosis resistance in WPE1-NB26 cells, we compared miRNA expression profiles between WPE1NA22 and WPE1-NB26 cells, using the mirVana miRNA Bioarray. Among the 471 human miRNAs examined, five miRNAs were found to be decreased and five miRNAs were found to be increased in WPE1-NB26 cells (Supplementary Table 1). Among these miRNAs, miR-205 and miR-31 were the most significantly downregulated ones in WPE1-NB26 cells, decreased by 93.1 and $77.1 \%$ of the levels in WPE1NA22 cells, respectively. The downregulation of miR-205 and miR-31 in WPE1-NB26 cells were confirmed by real-time PCR (Figure 2). We selected these two miRNAs to further study their roles in prostate cancer apoptosis.

miR-205 and miR-31 are downregulated in advanced prostate cancer cells. To determine whether miR-205 and miR-31 are downregulated in other cell lines derived from RWPE-1 and other malignant prostate cancer cell lines, we analyzed the expression levels of the two miRNAs by realtime PCR. As shown in Figure 2, the expression levels of miR-205 and miR-31 showed sequential decrease as the prostate cancer progresses from WPE1-NA22, WPE1-NB14, WPE1-NB11, to WPE1-NB26, in the order of increasing malignancy. ${ }^{24}$ Furthermore shown in Figure 2, miR-205 and a
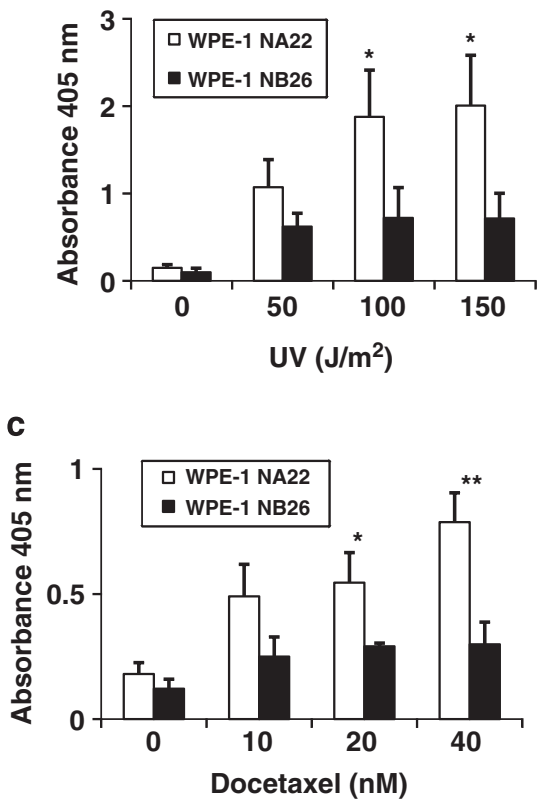

b

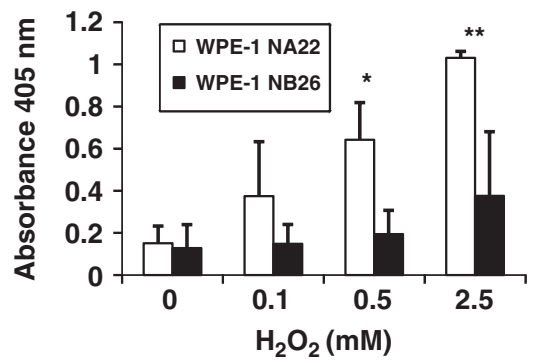

d

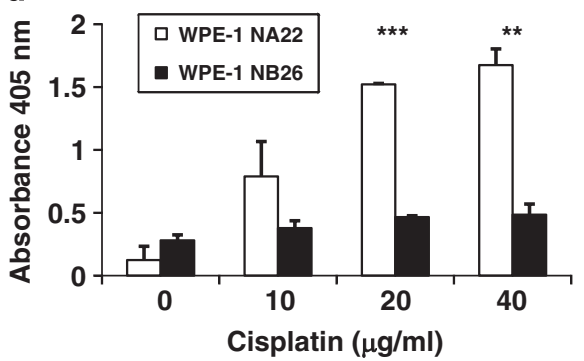

Figure 1 WPE1-NB26 cells are resistant to apoptosis. WPE1-NA22 and WPE1-NB26 cells were treated with the following apoptosis-inducing stimuli and apoptosis was analyzed using the Cell Death Detection Elisa kit as described in Materials and Methods section. (a) Cells were exposed to various doses of UV and then cultured for $24 \mathrm{~h}$. (b) Cells were treated with various doses of $\mathrm{H}_{2} \mathrm{O}_{2}$ for $24 \mathrm{~h}$. (c) Cells were exposed to various doses of Docetaxel for $24 \mathrm{~h}$. (d) Cells were treated with various doses of Cisplatin for $24 \mathrm{~h}$. All experiments have been repeated three times, data shown are mean values + S.D. $\left({ }^{\star} P<0.05,{ }^{\star \star} P<0.01,{ }^{\star \star \star} P<0.001\right)$ 

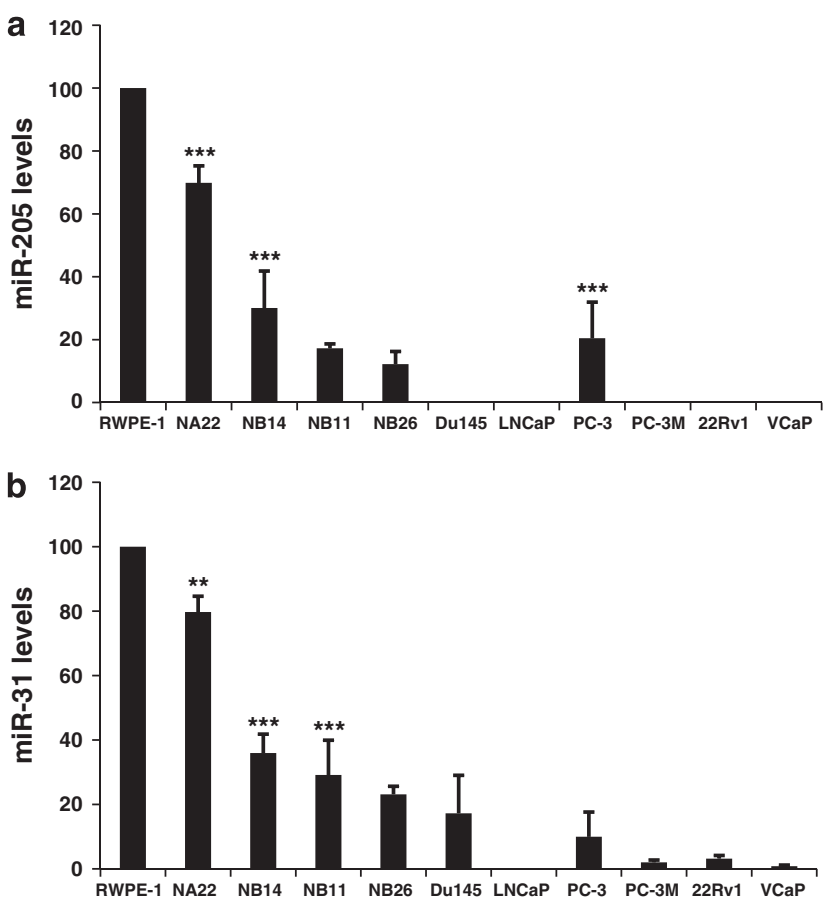

Figure 2 miR-205 and miR-31 are downregulated in advanced prostate cancer cells. Total RNA was isolated from the indicated cell lines and real-time PCR analysis was performed as described in Materials and Methods section to determine the expression of (a) miR-205 and (b) miR-31. The experiments have been repeated three times, data shown are mean values + S.D. $\left({ }^{\star \star} P<0.01\right.$ ${ }^{* * *} P<0.001$, compared with RWPE-1 cells)

miR-31 were significantly downregulated in PC-3, LNCaP, Du145, 22Rv1, VCaP, and PC-3M cells. We determined the responses of these cells to chemotherapy-induced apoptosis. As shown in Supplementary Figure 2A and 2B, while RWPE-1 cells were sensitive to Cisplatin- and Docetaxelinduced apoptosis, WPE1-NB14 and WPE1-NB11 cells were resistant to these drugs as the levels of miR-205 and miR-31 were decreased in these cells. Du145 and 22Rv1 cells were relatively resistant to Cisplatin (comparing with RWPE-1 and WPE1-NA22 cells, but to a lesser degree when comparing with PC-3 cells), and both Du145 and PC-3 cells were more resistant to Docetaxel than the 22Rv1 cells (Supplementary Figure 2C and 2D). As these cell lines all express low levels of miR-205 and miR-31, we reason that other drug-resistance mechanisms may contribute to the observed differences among their responses to these agents. However, despite the differences of their responses, they were all relatively resistant to Cisplatin and Docetaxel when compared with the RWPE-1 and WPE1-NA22 cells, which express higher levels of miR-205 and miR-31.

miR-205 targets Bcl-w and miR-31 targets E2F6. Using the computational methods available at http://www.microrna.org and miRBase (http://microrna.sanger.ac.uk/), we identified $\mathrm{Bcl}-\mathrm{w}$ as the potential target for miR-205, and E2F6 as the potential target for miR-31. Bcl-w is an antiapoptotic member of the Bcl-2 family proteins. ${ }^{25}$ E2F6 inhibits UV-and hypoxiainduced apoptosis. ${ }^{26,27}$ The antiapoptotic properties of Bcl-w and E2F6 make them interesting targets for miR-205 and
miR-31. The BCL2L2 mRNA (encoding Bcl-w) contains a $3^{\prime}$ untranslated region ( $3^{\prime} \cup T R$ ) sequence that is partially complementary to miR-205, and the E2F6 mRNA has a 3'UTR recognized by miR-31 (Figure $3 a$ ). When a cDNA fragment containing the $3^{\prime} U T R$ sequence of BCL2L2 was inserted downstream of the green fluorescent protein (GFP) gene in the pEGFP-C1 plasmid and the plasmid was transfected into WPE1-NB26 cells together with pcDNA6.2GW-miR-205 (to overexpress miR-205), GFP expression was reduced comparing with cells transfected with pEGFPBCL2L2-3'UTR and pcDNA6.2-GW-negative-control plasmids (Figure $3 \mathrm{~b}$, left). Similarly, miR-31 overexpressed from pcDNA6.2-GW-miR-31 decreased the expression of GFP when GFP was fused with E2F6 3'UTR (Figure 3b, right). The functions of the miRNAs were dependent on the miRNA binding sites within the BCL2L2 and E2F6 3'UTRs, as GFP expression was not reduced by the miRNAs when the binding sites were mutated (Figure $3 b$ ). The expression levels of the Bcl-w and E2F6 proteins were increased in WPE1-NB14, WPE1-NB11, and WPE1-NB26 cells comparing with the RWPE-1 and WPE1-NA22 cells (Figure $3 c$ ), agreeing with that miR-205 and miR-31 may regulate the expression of these two proteins. Bcl-w and E2F6 levels were also increased in Du145, LNCaP, PC-3, and 22Rv1 cells, comparing with RWPE-1 cells (Supplementary Figure 3A). To confirm that miR-205 regulates Bcl-w and miR-31 regulates E2F6, we overexpressed miR-205 and miR-31 in WPE1-NB26 cells using the pcDNA6.2-GW-miR miRNA expression vectors (Figure $3 \mathrm{~d}$, left). Overexpression of miR-205 and miR-31 downregulated Bcl-w and E2F6, respectively (Figure 3d, middle). Conversely, transfection of WPE1-NA22 cells with anti-miR miRNA inhibitors specific to miR-205 and miR-31 increased the protein levels of Bcl-w and E2F6, respectively (Figure 3d, right). The anti-miR-205 and anti-miR-31 inhibitors also blocked Docetaxel-induced apoptosis in WPE1-NA22 cells (Supplementary Figure 3B). In addition, we found that treatment with Cisplatin induced miR-205 and miR-31 expression in WPE1-NA22 cells and induced miR-31 expression in WPE1-NB26 cells (Supplementary Figure 4). Docetaxel had no significant effects on the expression of these miRNAs.

Bcl-w and E2F6 confer resistance to chemotherapyinduced apoptosis. We expressed Bcl-w and E2F6 exogenously to determine whether these proteins can contribute to apoptosis resistance in prostate cancer cells. To avoid toxicities associated with transient transfection, we created stable WPE1-NA22 cell lines expressing Bcl-w or E2F6. Overexpression of $\mathrm{Bcl}-\mathrm{w}$ or E2F6 was confirmed by western blotting (Figure $4 \mathrm{a}$ and $\mathrm{c}$ ). When treated with Docetaxel or Cisplatin, two agents used clinically to treat prostate cancer, cells stably expressing $\mathrm{Bcl}-\mathrm{w}$ were significantly resistant to drug-induced apoptosis, comparing with empty vectortransfected cells (Figure $4 a$ and b). Similarly, cells stably expressing E2F6 were also resistant to Docetaxel and Cisplatin (Figure 4c and d). Induction of apoptosis was also measured by annexin $\mathrm{V}$ staining (Supplementary Figure $5 \mathrm{~A}$ and $5 \mathrm{~B})$. Bcl-w- and E2F6-conferred resistance to druginduced apoptosis was confirmed by three independent 
a

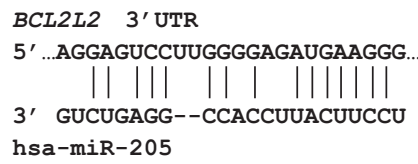

C

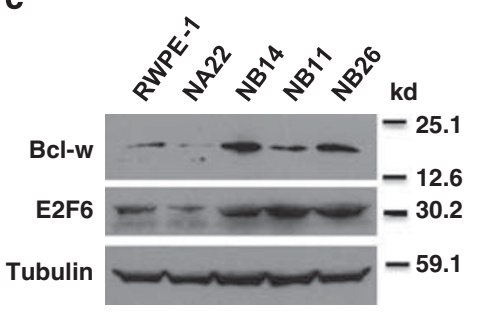

b

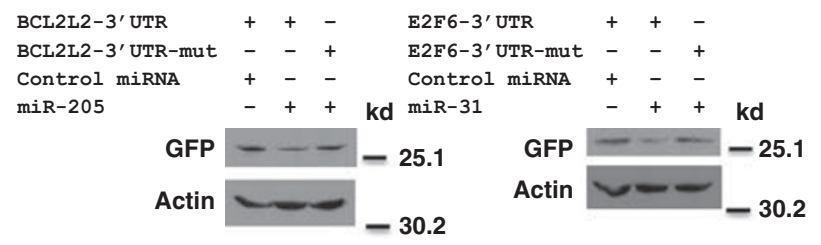

d
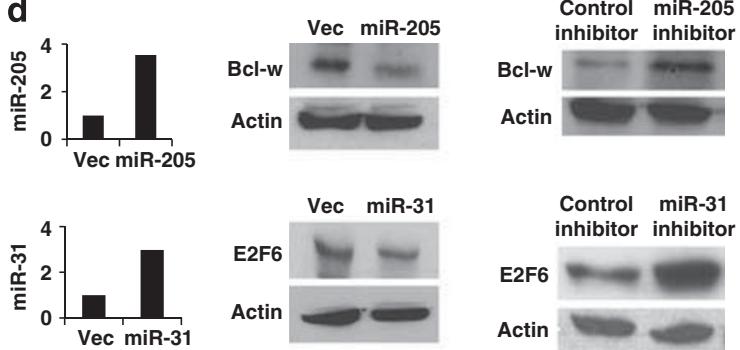

Figure 3 miR-205 targets Bcl-w and miR-31 targets E2F6. (a) Upper panel, predicted duplex formation between human BCL2L2 3'UTR and miR-205. Lower panel, predicted duplex formation between human E2F6 3'UTR and miR-31. (b) Left, WPE1-NB26 cells were transfected with GFP-BCL2L2 (3'UTR) or GFP-BCL2L2 (3'UTR)-mut plasmids together with plasmids expressing miR-205 or a negative control miRNA. Western blotting was performed with anti-GFP and anti-actin antibodies. Right, WPE1NB26 cells were transfected with GFP-E2F6 ( $3^{\prime}$ UTR) or GFP-E2F6 ( $3^{\prime}$ UTR)-mut plasmids together with plasmids expressing miR-31 or a negative control miRNA. Western blotting was performed with anti-GFP and anti-actin antibodies. (c) Proteins were isolated from the indicated cell lines, western blots were done using indicated antibodies. (d) Left, WPE1-NB26 cells were transfected with pcDNA6.2-GW/EmGFP-miR-negative control or pcDNA6.2-GW/EmGFP-miR205, or pcDNA6.2-GW/EmGFP-miR31 plasmids. Expression of miRNAs was confirmed by real-time PCR. At $48 \mathrm{~h}$ after transfection, cell lysates were analyzed by western blots with the indicated antibodies (Middle). Right, WPE1-NA22 cells were transfected with synthetic miRNA inhibitors specific to miR-205 or miR-31, or the negative control inhibitor. At $48 \mathrm{~h}$ after transfection, cell lysates were analyzed by western blots with the indicated antibodies. Representative images of three independent experiments were shown

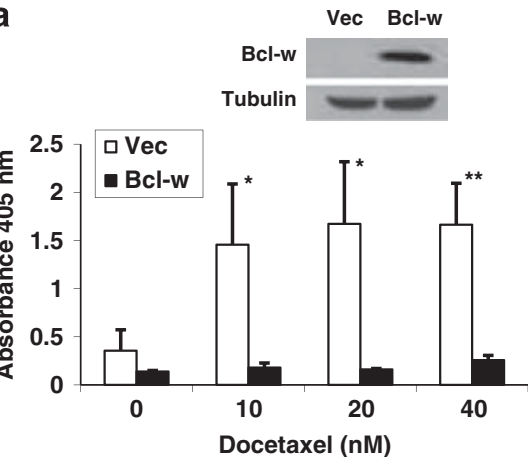

b

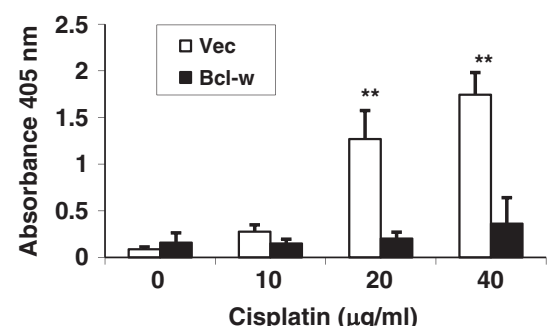

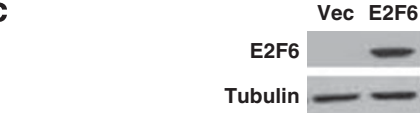

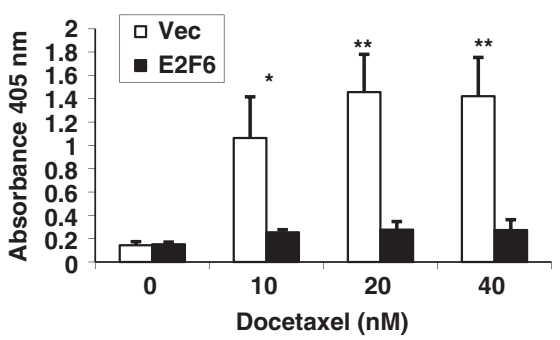

d

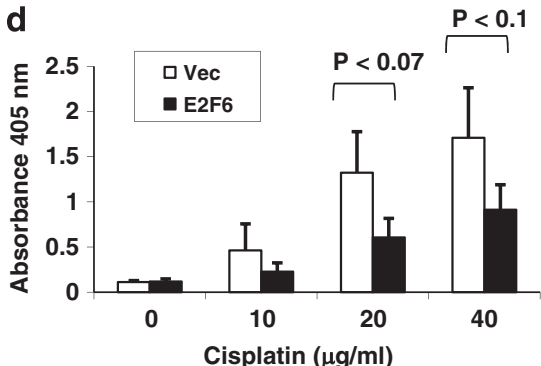

Figure 4 Bcl-w and E2F6 confer resistance to chemotherapy-induced apoptosis. (a) WPE1-NA22 cell line stably expressing Bcl-w was established and Bcl-w expression was confirmed by western blot. Empty vector transfected or Bcl-w stable expressing cells were treated with various doses of Docetaxel for $24 \mathrm{~h}$, and apoptosis was measured by Cell Death Detection Elisa analysis as described in Materials and Methods section. (b) Empty vector control or Bcl-w stable expressing WPE1-NA22 cells were treated with various doses of Cisplatin for $24 \mathrm{~h}$, and apoptosis was detected as above. (c) WPE1-NA22 cell line stably expressing E2F6 was established and E2F6 expression was confirmed by western blot. Empty vector transfected or E2F6 stable expressing cells were treated with various doses of Docetaxel for $24 \mathrm{~h}$, and apoptosis was measured as above. (d) Empty vector control or E2F6 stable expressing WPE1-NA22 cells were treated with various doses of Cisplatin for $24 \mathrm{~h}$, and apoptosis was detected as above. The experiments have been repeated three times, data shown are mean values + S.D. $\left({ }^{\star} P<0.05,{ }^{\star \star} P<0.01\right)$ 
clones of Bcl-w or E2F6 stable expressing cell lines (Supplementary Figure 6A and 6B).

miR-205 and miR-31 sensitize prostate cancer cells to chemotherapy-induced apoptosis. To determine the effects of miR-205 and miR-31 on chemotherapy-induced apoptosis in prostate cancer cells, we established WPE1NB26 cell lines stably expressing miR-205 or miR-31. As shown in Figure $5 \mathrm{a}$ and $\mathrm{c}$, stable expression of miR-205 and miR-31 was confirmed by real-time PCR analysis. Overexpression of miR-205 sensitized WPE1-NB26 cells to apoptosis induced by both Docetaxel and Cisplatin (Figure $5 \mathrm{a}$ and b). Similarly, stable expression of miR-31 also sensitized WPE1-NB26 cells to apoptosis following exposure to these agents (Figure $5 c$ and $d$ ). Differences in apoptotic responses were also measured by annexin $\mathrm{V}$ staining (Supplementary Figure 5C and 5D). Sensitization to drug-induced apoptosis by miR-205 and miR-31 was confirmed using three independent clones of miR-205 or miR-31 stable expressing cell lines (Supplementary Figure $6 \mathrm{C}$ and 6D).

Promoter hypermethylation causes the downregulation of miR-205 in advanced prostate cancer. To determine the mechanism of how miR-205 and miR-31 were downregulated in prostate cancer cells, we explored whether epigenetic silencing by promoter hypermethylation had a role in their expression. We first performed rapid amplification of $5^{\prime}$ complementary DNA ends ( $5^{\prime}$ RACE) experiments to identify the transcription start site and the promoter for the miR-205 gene. The gene encoding miR-205 is located on chromosome 1. The transcription start site for miR-205 was identified and a $\mathrm{CpG}$ island was found in the promoter region of the miR-205 gene about 300 base pairs in front of the first exon (Figure 6a). The CpG island is potential site to be modified by methylation. To determine whether the miR-205 promoter is methylated in prostate cancer cells, we isolated genomic DNAs from various prostate cancer cell lines, treated the DNA with sodium bisulfate, and performed methylation-specific PCR using appropriate primers that could detect the methylated or unmethylated promoter DNAs. As shown in Figure 6b, methylation of the miR-205 CpG island was detected in Du145, PC-3, LNCaP, 22Rv1, WPE1-NB11, WPE1-NB14, and PC-3M cells, but not in WPE1-NA22 and WPE1-NB26 cells. When PC-3M cells were treated with 5-aza-2'-deoxycytidine, an inhibitor of the DNA methyltransferase, the level of miR-205 was increased (Figure 6c, top). Conversely, the protein level of Bcl-w was decreased after treatment with 5-aza-2'-deoxycytidine (Figure 6c, bottom). Transfection with anti-miR-205 inhibitor blocked the decrease of Bcl-w caused by 5-aza-2'-deoxycytidine (Figure 6c, bottom). We then tested whether 5-aza$2^{\prime}$-deoxycytidine can potentiate Cisplatin-induced apoptosis in PC-3M cell. As shown in Figure $6 \mathrm{~d}$ and e, pre-treatment
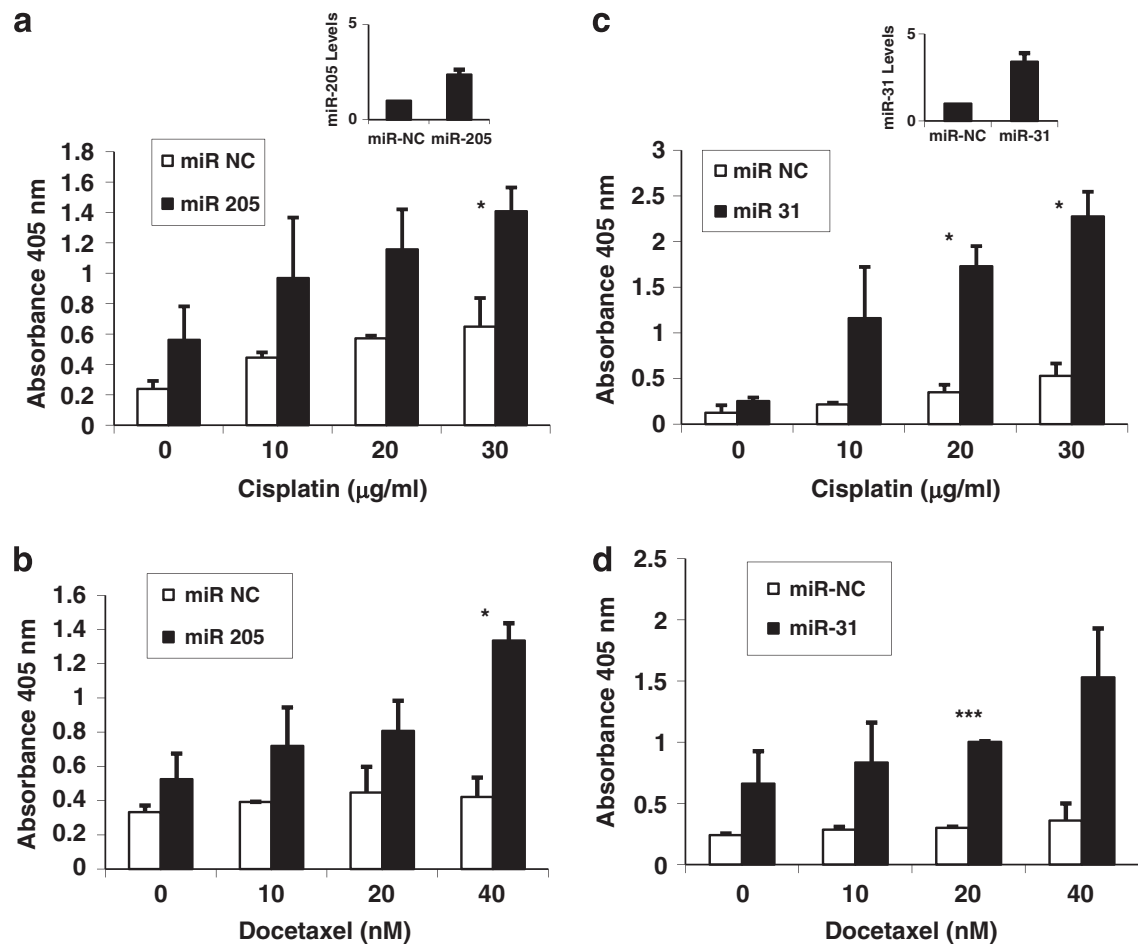

Figure 5 miR-205 and miR-31 sensitize WPE1-NB26 cells to chemotherapy-induced apoptosis. (a) WPE1-NB26 cell line stably expressing miR-205 was established and miR-205 expression was confirmed by real-time PCR. Negative control miRNA or miR-205 expressing cells were treated with various doses of Docetaxel for $24 \mathrm{~h}$, and apoptosis was measured by Cell Death Detection Elisa analysis as described in Materials and Methods section. (b) Negative control or miR-205 expressing WPE1-NB26 cells were treated with various doses of Cisplatin for $24 \mathrm{~h}$, and apoptosis was detected as above. (c) WPE1-NB26 cell line stably expressing miR-31 was established and miR-31 expression was confirmed by real-time PCR. Negative control miRNA or miR-31 expressing cells were treated with various doses of Docetaxel for $24 \mathrm{~h}$, and apoptosis was measured as above. (d) Negative control miRNA or miR-31 expressing WPE1-NB26 cells were treated with various doses of Cisplatin for $24 \mathrm{~h}$, and apoptosis was detected as above. The experiments have been repeated three times, data shown are mean values + S.D. $\left({ }^{\star} P<0.05,{ }^{* \star \star} P<0.001\right)$ 
a

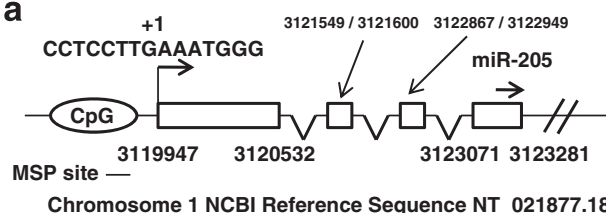

b

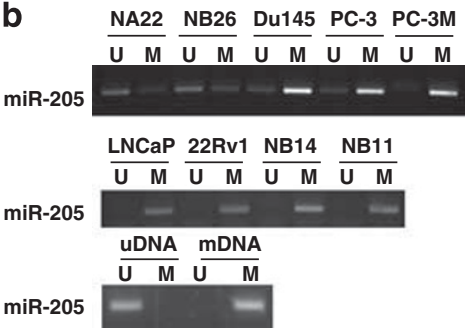

Figure 6 Promoter hypermethylation suppresses the expression of miR-205 in advanced prostate cancer cells. (a) The putative transcription start site (indicated by +1 ) and genomic sequences of the miR-205 gene is shown. The mature miR-205 sequence is indicated by ' $\rightarrow$ '. The DNA fragment covered by methylation-specific PCR is indicated by '-'. The locations of the first four exons on the chromosomes were indicated. (b) Methylation-specific PCR was performed as described in Materials and Methods section, using genomic DNA isolated from the indicated cells lines. M, methylated DNA; U, unmethylated DNA. Bottom, control PCR was done using Qiagen EpiTect unmethylated (uDNA) and methylated DNA (mDNA) as templates. (c) Top, PC-3 M cells were treated with $5 \mu \mathrm{M}$ 5-aza-2'-deoxycytidine for $24 \mathrm{~h}$, and then cultured in drug-free media for 7 days. Total RNA was isolated and real-time PCR was performed as described in Materials and Methods section. Bottom, PC-3 M cells were treated with $5 \mu \mathrm{M}$ 5-aza-2'-deoxycytidine for $24 \mathrm{~h}$, followed with or without transfection of anti-miR-205 inhibitor, and cultured in drug-free media for 7 days, western blot was performed using the indicated antibodies. (d and e) PC-3 M cells were treated with $5 \mu \mathrm{M} \mathrm{5-aza-2'-deoxycytidine} \mathrm{for} 24 \mathrm{~h}$ and then cultured in drug-free media for 2 days with or without transfection of anti-miR-205 inhibitor, followed by various doses of Cisplatin for $24 \mathrm{~h}$. Apoptosis was measured by Cell Death Detection Elisa analysis (d) or annexin V staining (e). The experiments have been repeated three times, data shown are mean values + S.D. $\left({ }^{*} P<0.05\right)$

with 5-aza-2'-deoxycytidine significantly enhanced Cisplatininduced apoptosis, possibly through activating miR-205 expression. The effects of 5-aza-2'-deoxycytidine were blocked by synthetic anti-miR-205 inhibitor. We also cloned the miR-31 gene promoter. However, the results of miR-31 promoter methylation studies were inconclusive. While 5-aza-2'-deoxycytidine induced a modest increase of miR-31 expression (about 50\% increase) in PC-3M cells, it failed to induce miR-31 expression in other prostate cancer cell lines (data not shown).

\section{Discussion}

MicroRNAs have been indicated to have important roles in the regulation of cancer cell functions including differentiation, proliferation, apoptosis, and metastasis. ${ }^{28,29}$ In this study, we have demonstrated that miR-205 and miR-31 regulate apoptosis in prostate cancer cells by targeting antiapoptotic proteins $\mathrm{Bcl}-\mathrm{w}$ and E2F6. In prostate cancer cell lines derived from advanced metastatic cancers, miR-205 and miR-31 are downregulated to contribute to the resistance to chemotherapy-induced apoptosis. Thus, our data have established a mechanistic basis to explain the resistance to chemotherapy often observed in advanced prostate cancers. This study has also identified miR-205 and miR-31 as key targets to improve prostate cancer response to chemotherapy.

It is known that miRNAs can target many mRNAs and Bcl-w and E2F6 are not the sole targets of miR-205 and miR-31. Other genes targeted by these miRNAs could also have a role in prostate cancer apoptosis. However, as shown by our studies using Bcl-w and E2F6 stable expressing cell lines, these two proteins confer strong resistance to chemotherapyinduced apoptosis, supporting a key role in mediating the effects of miR-205 and miR-31 on apoptosis. Bcl-w is a member of the Bcl-2 family proteins ${ }^{25}$ and inhibits apoptosis by blocking the intrinsic pathway of apoptosis. ${ }^{30}$ E2F6 is a strong transcription repressor and suppresses both UV- and hypoxia-induced apoptosis. ${ }^{26,27}$ It is likely that E2F6 blocks apoptosis by suppressing the expression of certain pro-apoptotic genes. The individual contribution of miR-205 and miR-31 (as well as Bcl-w and E2F6) to apoptotic response in prostate cancer cells remains to be established. From the data shown in our studies using the stable cell lines (Figures 4 and 5), overexpression of miR-31 alone seems to be sufficient to induce apoptosis in WPE1-NB26 cells at comparable level to WPE1-NA22 cells. In addition, although Bcl-w blocks Cisplatin-induced apoptosis more effectively than E2F6 in the overexpression experiments, miR-31 seems to be more efficient in sensitizing WPE1-NB26 cells to Cisplatin. These observations could be explained by the possible suppression of other target genes with miR-31 overexpression, which may contribute to the increased induction of apoptosis in response to Cisplatin.

Although we found $\mathrm{CpG}$ island methylation in the promoter of miR-205 in various advanced prostate cancer cell lines, we did not detect DNA methylation of the miR-205 promoter in WPE1-NB26 cells. Thus, other mechanisms may be behind the downregulation of miR-205 in the WPE1-NB26 cells. 
Certain types of histone modifications can function as a silencing mechanism independent of DNA methylation. For example, it is found that histone H3K27 trimethylation is responsible for epigenetic silencing of miR-22 independent of promoter methylation. ${ }^{31}$ Alternatively, downregulation of the transcription factors that are required for miR-205 expression may contribute to the silencing of the miRNA in WPE1-NB26 cells. Whether DNA methylation contributes to the downregulation of miR-31 in prostate cancer is not clear. Factors discussed above may have a role in the silencing of miR-31. In addition, mutations or deletions of the miR-31 gene may also be key reasons behind the downregulation of the miRNA in prostate cancer.

Our data indicate that downregulation of miR-205 and miR-31 may represent a key step in prostate cancer progression into the more aggressive and chemo-resistant cancers. Strategies to reactivating the expression of these miRNAs (such as the use of 5-aza-2'-deoxycytidine in our study) may prove successful to overcome resistance to chemotherapy in advanced prostate cancers.

\section{Materials and Methods}

Cells and transfection. The cell lines RWPE-1, WPE1-NA22, WPE1-NB14, WPE1-NB11, WPE1-NB26, PC-3, LNCaP, 22Rv1, VCaP, and Du145 were purchased from American Type Culture Collection (Manassas, VA, USA). The RWPE-1, WPE1-NA22, WPE1-NB14, WPE1-NB11, and WPE1-NB26 cells were cultured in Keratinocyte Serum Free Medium (K-SFM, Invitrogen, Carlsbad, CA, USA), supplemented with bovine pituitary extract and human recombinant epidermal growth factor. PC-3, LNCaP, 22Rv1, VCaP, and Du145 cells were cultured in RPMI1640 media containing 10\% FBS. For transient transfection, plasmids were transfected into cells using LipofectaminePlus Reagent (Invitrogen) following the manufacturer's protocol. miRNA mimics and inhibitors were transfected into cells using X-treme GENE siRNA transfection reagent (Roche, Indianapolis, IN, USA) following the manufacturer's protocol.

Drugs and chemicals. Hydrogen peroxide, Docetaxel, and Cisplatin were purchased from Sigma (St. Louis, MO, USA).

Plasmids construction, miRNA mimics and inhibitors. The $3^{\prime} U T R$ CDNA fragments of BCL2L2 and E2F6 genes were obtained by PCR using EST clones as templates. The $3^{\prime} U T R$ CDNAs were inserted into pEGFP-C1 plasmid at the $3^{\prime}$ end of the EGFP cDNA (Xho I and BamH I). The pEGFP-C1-3'UTR plasmids containing mutations within the miRNA recognition sites (AAGG to TTCC mutation for BCL2L2 $3^{\prime} U T R$, and TTGC to AACG mutation for E2F6 $3^{\prime} U T R$ ) were created by PCR using the QuickChange II site-directed mutagenesis kit (Stratagene, Santa Clara, CA, USA), following the supplied protocol. The full-length BCL2L2 and E2F6 cDNAs were obtained by PCR using EST clones as templates and constructed into pcDNA3-HA vector for creation of the stable expression cell lines. To construct miRNA expression vectors, double-strand oligoes containing pre-miR-205 or premiR-31 were cloned into pcDNA6.2-GW/EmGFP-miR plasmids (Invitrogen) by directional cloning. For co-transfection of pEGFP-C1-3'UTR and miRNA expression vectors, EmGFP was removed using Dra I digestion to create pcDNA6.2-GWmiR-205 and pcDNA6.2-GW-miR-31. miRIDIAN miR-205 and miR-31 mimics (Cat\# C-300564-05 and C-300507-05, respectively) and negative control miRNA (Cat\# CN-001000-01-05) were purchased from Dharmacon (Lafayette, CO, USA). Anti-miR miRNA inhibitors for miR-205 and miR-31 (ID AM11015 and AM11465, respectively) were purchased from Ambion (Austin, TX, USA).

Creation of stable expression cell lines. Stable cell lines expressing Bcl-w or E2F6 were established by transfection of WPE1-NA22 cells with pcDNA3HA-BCL2L2 or pcDNA3-HA-E2F6. Colonies were selected in G418-containing media and cloned with cloning cylinders. Overexpression of Bcl-w or E2F6 was determined by western blotting with anti-HA antibodies. Stable cell lines expressing miR-205 or miR-31 were established by transfection of WPE1-NB26 cells with Block-iT Pol II miR expression vectors: pcDNA6.2-GW/EmGFP-miR-205 or
pcDNA6.2-GW/EmGFP-miR-31. Colonies were selected in Blasticidin-containing media and cloned with cloning cylinders. Overexpression of miR-205 and miR-31 were determined by real-time PCR analysis.

Western blot analysis. Cells were lysed in RIPA buffer (1\% NP-40, $0.5 \%$ sodium deoxycholate, $0.1 \%$ SDS in PBS). Complete protease inhibitor cocktail (Roche) was added to lysis buffer before use. Protein concentration was determined by Bio-Rad DC protein assay (Bio-Rad, Hercules, CA, USA). Protein samples were subjected to SDS-PAGE and transferred to nitrocellulose membrane. The membrane was blocked in $5 \%$ non-fat milk in PBS overnight and incubated with primary antibody and subsequently with appropriate horseradish peroxidaseconjugated secondary antibody. Signals were developed with ECL reagents (Pierce, Rockford, IL, USA) and exposure to X-ray films. Anti-Bcl-w polyclonal antibody was purchased from Stressgen or Enzo (Plymouth Meeting, PA, USA). Anti- $\beta$-tubulin and anti-E2F6 antibodies were purchased from Santa Cruz Biotechnology (Santa Cruz, CA, USA).

Real-time PCR. The miRNA expression was measured by real-time PCR using TaqMan MicroRNA assays (Cat \# TM509 for miR205, TM1100 for miR-31) from Applied Biosystems (Foster City, CA, USA). Total RNA was isolated from cells using mirVana miRNA Isolation Kit (Ambion). A measure of $5 \mu \mathrm{g}$ of total RNA was used in reverse transcription reaction. The CDNAs were used as templates to perform PCR on an Applied Biosystems 7500 Real-time PCR System following the manufacturer's protocol. Relative miRNA expression levels were calculated using 18S RNA as reference.

miRNA expression profiling. Total RNAs were isolated from WPE1-NA22 and WPE1-NB26 cells using the mirVana miRNA Isolation Kit (Ambion). miRNAs were further concentrated with the flashPAGE Fractionator System (Ambion). The miRNAs from WPE1-NA22 and WPE1-NB26 cells were labeled with the mirVana miRNA Labeling Kit (Ambion) with Cy3 or Cy5 Post Labeling Reactive Dye (Amersham Biosciences, Piscataway, NJ, USA), respectively. The probes were then hybridized to the mirVana miRNA Bioarrays (Ambion) overnight. The array slides were scanned with an Aron 4000B Genepix Microarray Scanner and analyzed with the Genepix software (Molecular Devices, Sunnyvale, CA, USA).

5' $^{\prime}$ RACE. The transcription start site of miR-205 pri-miRNA was identified by $5^{\prime}$ RACE experiments with FirstChoice RLM-RACE kit from Ambion, using total RNA isolated from WPE1-NA22 cells as template.

Methylation-specific PCR. The bisulfate conversion of cytosine to uracil of the genomic DNA was performed with the EZ DNA Methylation-Gold kit from Zymo Research (Orange, CA, USA). Methylation-specific PCR was done with the following primers: miR-205 methylated forward, 5'-GAGTTTAAGTTGCGT ATGGAAGC-3', reverse, 5'-AAAACAAATATTTCTTTTATAATCCGAA-3'. miR-205 unmethylated forward, $5^{\prime}$-GGAGTTTAAGTTGTGTATGGAAGTG-3', reverse, $5^{\prime}$-AA AACAAATATTTCTTTTATAATCCAAA-3'. Bisulfite converted methylated and unmethylated human control DNAs (EpiTect PCR Control DNA Set from Qiagen, Valencia, CA, USA) were used as positive control.

Detection of apoptosis. The Cell Death Detection Elisa kit (Roche) was used to detect apoptosis following the manufacturer's protocol. This assay determines apoptosis by measuring mono and oligonucleosomes in the lysates of apoptotic cells. The cell lysates were placed into a streptavidin-coated microplate and incubated with a mixture of anti-histone-biotin and anti-DNA-peroxidase. The amount of peroxidase retained in the immunocomplex was photometrically determined with ABTS as the substrate. Absorbance was measured at $405 \mathrm{~nm}$. For annexin V staining, cells were washed with cold phosphate-buffered saline (PBS) and diluted in annexin-binding buffer at $1 \times 10^{6} \mathrm{cells} / \mathrm{ml}$. A volume of $5 \mu \mathrm{l}$ of annexin V fluorescein conjugate (Invitrogen) was added to each $100 \mu \mathrm{l}$ of cell suspension and cells were incubated at room temperature for $15 \mathrm{~min}$. After incubation, cells were analyzed by flow cytometry.

Statistical analysis. Differences between the mean values were analyzed for significance using the unpaired two-tailed Student's test for independent samples; $P \leq 0.05$ was considered to be statistically significant.

\section{Conflict of interest}

The authors declare no conflict of interest. 
Acknowledgements. We thank Jodie Haring for providing help with real-time PCR studies. This research was supported by NIH Grant CA130062 and RR015566.

1. McConkey DJ, Greene G, Pettaway CA. Apoptosis resistance increases with metastatic potential in cells of the human LNCaP prostate carcinoma line. Cancer Res 1996; 56: 5594-5599.

2. Van Brussel JP, Jan Van Steenbrugge G, Van Krimpen C, Bogdanowicz JF, Van Der Kwast $\mathrm{TH}$, Schroder $\mathrm{FH}$ et al. Expression of multidrug resistance related proteins and proliferative activity is increased in advanced clinical prostate cancer. J Urol 2001; 165 : 130-135.

3. Berezovskaya O, Schimmer AD, Glinskii AB, Pinilla C, Hoffman RM, Reed JC et al. Increased expression of apoptosis inhibitor protein XIAP contributes to anoikis resistance of circulating human prostate cancer metastasis precursor cells. Cancer Res 2005; 65 2378-2386.

4. Zhang M, Latham DE, Delaney MA, Chakravarti A. Survivin mediates resistance to antiandrogen therapy in prostate cancer. Oncogene 2005; 24: 2474-2482.

5. Hadaschik BA, Sowery RD, Gleave ME. Novel targets and approaches in advanced prostate cancer. Curr Opin Urol 2007; 17: 182-187.

6. Bartel DP. MicroRNAs: target recognition and regulatory functions. Cell 2009; 136: 215-233.

7. Bartel DP. MicroRNAs: genomics, biogenesis, mechanism, and function. Cell 2004; 116 : 281-297.

8. Kota J, Chivukula RR, O'Donnell KA, Wentzel EA, Montgomery CL, Hwang HW et al. Therapeutic microRNA delivery suppresses tumorigenesis in a murine liver cancer model. Cell 2009; 137: 1005-1017.

9. Chen CZ, Li L, Lodish HF, Bartel DP. MicroRNAs modulate hematopoietic lineage differentiation. Science 2004; 303: 83-86.

10. Zhang J, Jima DD, Jacobs C, Fischer R, Gottwein E, Huang $G$ et al. Patterns of microRNA expression characterize stages of human B-cell differentiation. Blood 2009; 113: 4586-4594.

11. Poy MN, Eliasson L, Krutzfeldt J, Kuwajima S, Ma X, Macdonald PE et al. A pancreatic islet-specific microRNA regulates insulin secretion. Nature 2004; 432: 226-230.

12. Ma L, Teruya-Feldstein J, Weinberg RA. Tumour invasion and metastasis initiated by microRNA-10b in breast cancer. Nature 2007; 449: 682-688.

13. Hermeking H. The miR-34 family in cancer and apoptosis. Cell Death Differ 2009; 17: 193-199.

14. Ellis L, Atadja PW, Johnstone RW. Epigenetics in cancer: targeting chromatin modifications. Mol Cancer Ther 2009; 8: 1409-1420.

15. McCabe MT, Brandes JC, Vertino PM. Cancer DNA methylation: molecular mechanisms and clinical implications. Clin Cancer Res 2009; 15: 3927-3937.

16. Bastian PJ, Yegnasubramanian S, Palapattu GS, Rogers CG, Lin X, De Marzo AM et al. Molecular biomarker in prostate cancer: the role of $\mathrm{CpG}$ island hypermethylation. Eur Urol 2004; 46: 698-708

17. Yegnasubramanian S, Kowalski J, Gonzalgo ML, Zahurak M, Piantadosi S, Walsh PC et al. Hypermethylation of $\mathrm{CpG}$ islands in primary and metastatic human prostate cancer. Cancer Res 2004; 64: 1975-1986.
18. Lujambio A, Ropero S, Ballestar E, Fraga MF, Cerrato C, Setien F et al. Genetic unmasking of an epigenetically silenced microRNA in human cancer cells. Cancer Res 2007; 67: 1424-1429.

19. Lujambio A, Calin GA, Villanueva A, Ropero S, Sanchez-Cespedes M, Blanco D et al. A microRNA DNA methylation signature for human cancer metastasis. Proc Natl Acad Sci USA 2008; 105: 13556-13561.

20. Agirre X, Vilas-Zornoza A, Jimenez-Velasco A, Martin-Subero Jl, Cordeu L, Garate L et al. Epigenetic silencing of the tumor suppressor microRNA Hsa-miR-124a regulates CDK6 expression and confers a poor prognosis in acute lymphoblastic leukemia. Cancer Res 2009; 69: 4443-4453.

21. Bandres E, Agirre X, Bitarte N, Ramirez N, Zarate R, Roman-Gomez J et al. Epigenetic regulation of microRNA expression in colorectal cancer. Int $J$ Cancer 2009; 125: 2737-2743.

22. Hoffman AE, Zheng T, Yi C, Leaderer D, Weidhaas J, Slack F et al. microRNA miR-196a-2 and breast cancer: a genetic and epigenetic association study and functional analysis. Cancer Res 2009; 69: 5970-5977.

23. Pallasch $\mathrm{CP}$, Patz $\mathrm{M}$, Park YJ, Hagist $\mathrm{S}$, Eggle D, Claus $\mathrm{R}$ et al. miRNA deregulation by epigenetic silencing disrupts suppression of the oncogene PLAG1 in chronic lymphocytic leukemia. Blood 2009; 114: 3255-3264.

24. Webber MM, Quader ST, Kleinman HK, Bello-DeOcampo D, Storto PD, Bice G et al. Human cell lines as an in vitrolin vivo model for prostate carcinogenesis and progression. Prostate 2001; 47: 1-13.

25. Gibson L, Holmgreen SP, Huang DC, Bernard O, Copeland NG, Jenkins NA et al. bcl-w, a novel member of the bcl-2 family, promotes cell survival. Oncogene 1996; 13: 665-675.

26. Yang WW, Wang ZH, Zhu Y, Yang HT. E2F6 negatively regulates ultraviolet-induced apoptosis via modulation of BRCA1. Cell Death Differ 2007; 14: 807-817.

27. Yang WW, Shu B, Zhu Y, Yang HT. E2F6 inhibits cobalt chloride-mimetic hypoxia-induced apoptosis through E2F1. Mol Biol Cell 2008; 19: 3691-3700.

28. Lujambio A, Esteller M. How epigenetics can explain human metastasis: a new role for microRNAs. Cell Cycle 2009; 8: 377-382.

29. Medina PP, Slack FJ. microRNAs and cancer: an overview. Cell Cycle 2008; 7: 2485-2492.

30. Wang X. The expanding role of mitochondria in apoptosis. Genes Dev 2001; 15: 2922-2933.

31. Li X, Liu J, Zhou R, Huang S, Chen XM. Gene silencing of MIR22 in acute lymphoblastic leukaemia involves histone modifications independent of promoter DNA methylation. Br J Haematol 2010; 148: 69-79.

Cell Death and Disease is an open-access journal published by Nature Publishing Group. This work is licensed under the Creative Commons Attribution-Noncommercial-No Derivative Works 3.0 Unported License. To view a copy of this license, visit http://creativecommons.org/licenses/by-nc-nd/3.0/

\section{Supplementary Information accompanies the paper on Cell Death and Disease website (http://www.nature.com/cddis)}

